Mots-clés

Dromadaire - Jeune animal - Mortalité Maroc.

\title{
Etude de la mortalité des chamelons au Sud du Maroc: enquête rétrospective
}

\author{
M. Bengoumi ${ }^{1}$ E.B. Gandega ${ }^{2}$ A. El Abrak ${ }^{3}$ \\ J. Berrada ${ }^{1}$ B. Faye ${ }^{4}$
}

\begin{abstract}
Résumé
Une enquête rétrospective a été réalisée entre janvier et août 1999 dans les provinces d'Oued Eddahab, Laâyoune, Guelmim, Tata, Essaouira, Zagora, O uarzazate, Errachidia et Figuig. Elle a concerné 252 troupeaux camelins, soit 15 p. 100 des effectifs recensés dans ces régions. La mortalité périnatale du chamelon reste une contrainte majeure au développement de l'élevage camelin avec en moyenne 20,2 p. 100 de la mortalité (16,4 p. 100 en 1996 et 22,9 p. 100 en 1997). L'âge de la mort se situait entre 0 et 6 mois dans plus de 80 p. 100 des cas. Les premières causes de mortalité citées par les éleveurs étaient la diarrhée (72 p. 100), puis, très loin derrière, l'insuffisance alimentaire (9 p. 100), la mortinatalité (6 p. 100) et les abcès (5 p. 100), avec de fortes variations selon les provinces. La typologie des élevages a permis de distinguer quatre classes selon le profil sanitaire et cinq classes selon les pratiques d'élevage. II existait de fortes corrélations entre la mortalité des chamelons et certaines pathologies des dromadaires adultes, notamment le syndrome respiratoire, la variole, la gale-teigne et d'autres pathologies. La forte homogénéité des modes de conduite n'a pas permis de mettre en évidence des corrélations entre la mortalité et les pratiques d'élevage qui jouaient probablement un rôle important.
\end{abstract}

\section{INTRO DUCTION}

La mortalité des chamelons est une contrainte majeure à la productivité du cheptel camelin en élevage extensif. En Ethiopie, des taux de 31 à 59 p. 100 ont été rapportés (4) ; Richard notait un taux de mortalité de 30 à 40 p. 100 dans la tranche d'âge de 0 à 6 mois (7). Burgmeister a noté un taux de mortalité de 26 p. 100 en Tunisie (3). Wilson estime que la mortalité néonatale jusqu'à un an est voisine de 50 p. 100 et considère que 30 p. 100 est un taux normal de mortalité (10). Richard et coll. ont observé un taux de mortalité au Niger de 44,6 p. 100 dans la tranche d'âge de 0 à 6 mois (8). Toujours au Niger, Saley a rapporté des taux de 10 à 25 p. 100 entre 0 et 6 mois et de 12 à 18 p. 100 entre 6 et 12 mois (9). Dans la province de Laâyoune, la mortalité varie, suivant les conditions climatiques et les troupeaux, de 7 à 60 p. 100 avec une moyenne de 22 à 25 p. 100 en 1995 et de 5 à 19 p. 100 en 1996 (1).

Dans les conditions actuelles, il semble que l'identification des causes de la mortalité des chamelons soit posée comme une condition au développement de l'élevage camelin. Devant une

1. Institut agronomique et vétérinaire Hassan II, BP 6202, Rabat-Instituts, Maroc

2. Cnerv, BP 167, Nouakchott, Mauritanie

3. Lnez, BP 6472, Rabat-Instituts, Maroc

4. Cirad-emvt, Productions animales, 34398 Montpellier Cedex 5, France pathologie multifactorielle, l'approche écopathologique est la démarche la mieux adaptée à l'identification de l'étiologie et des facteurs de risques.

\section{MATERIEL ET METHODES}

L'étude a été réalisée dans les provinces sahariennes du Maroc (Errachidia, Essaouira, Figuig, Guelmim, Laâyoune, Oued Eddahab, Ouarzazate, Tata et Zagora). C'est la zone d'élevage camelin du pays. Même si dans la zone d'Essaouira les troupeaux sont en général de petite taille, la diversité des systèmes justifie le choix de cette province pour cette étude. Les éleveurs ont été choisis de façon aléatoire, soit aux points d'eau, soit directement dans les douars, notamment lors des jours de grands rassemblements (marchés par exemple). L'ensemble des zones à camelins des provinces choisies a été couvert par l'enquête qui s'est déroulée entre janvier et août 1999.

Le questionnaire utilisé comprenait trois parties principales ainsi qu'une annexe sur les pathologies les plus fréquentes apparues dans le troupeau pendant les trois dernières années. L'unité statistique était représentée par le troupeau, défini comme un groupe d'animaux conduits ensemble sur les mêmes parcours et bénéficiant de pratiques d'élevage similaires. 
Les questions se rapportaient à l'organisation des hommes autour de l'élevage camelin en général et du troupeau en particulier, notamment les pratiques zootechniques, les soins vétérinaires ainsi que les événements survenus pendant les trois dernières années. Les questions étaient regroupées en trois types d'informations correspondant aux trois grandes divisions du questionnaire. Dans la base de données, chaque enregistrement représentait un troupeau et, dans la plupart des cas, un éleveur seul ou associé. Le masque de saisie a été élaboré avec le concours de l'équipe de statisticiens et d'informaticiens du Cirad-emvt. La saisie des questionnaires remplis a été effectuée au fur et à mesure de leur arrivée, ainsi que sur le terrain pendant les enquêtes des provinces d'Ouarzazate et d'Errachidia. Pour des raisons pratiques de classification, une numérotation unique des questionnaires a été adoptée lors de la saisie (encadrée sur la première page du questionnaire).

Un premier tri des variables a été fait pour éliminer les données redondantes ou inintéressantes. Le recodage de variables continues en classes et la définition de modalités ont été effectués à partir des réponses saisies ; le regroupement de réponses proches a été effectué. De nouvelles variables ont été définies, permettant de mieux représenter l'information recueillie lors de l'enquête. Les variables pertinentes ont été retenues pour l'analyse multivariée.

La fréquence des réponses aux questions a permis l'analyse statistique des valeurs de chaque groupe de variables homogènes et la mise en évidence des caractéristiques de l'échantillon pour les questions se rapportant au système de production camelin : organisation des éleveurs, stratégies de déplacement, structure du troupeau, pratiques d'élevage, pathologie des dromadaires et mortalité des chamelons et leurs causes.

L'analyse des correspondances multiples (Acm) et une classification ascendante hiérarchique (Cah) sur les facteurs obtenus par l'Acm ont été effectuées sur le logiciel Winstat. Deux classifications des élevages ont été faites, l'une suivant le profil sanitaire des adultes et l'autre sur les pratiques d'élevage. Les corrélations entre variables ont été testées par la méthode du $\mathrm{Chi}^{2}$ après croisement des variables.

\section{RESU LTATS ET DISCU SSIO N}

Les résultats de ce travail ont été obtenus par des enquêtes de terrain effectuées en grande partie par des techniciens connaissant bien le système de production camelin au Maroc, mais qui n'étaient pas à l'abri de biais éventuels (uniformisation des réponses à certaines questions, interrogation des éleveurs les plus accessibles, nombre insuffisant d'éleveurs enquêtés...). Au-delà du modeste rapport présenté ici, ce travail a regroupé des chercheursenseignants dans différents domaines, des épidémiologistes et des praticiens vivant le quotidien du terrain, d'où des contributions diverses et enrichissantes.

L'importance de l'élevage camelin dans les provinces sahariennes du Maroc n'est plus à démontrer et la mobilisation autour de cet animal des zones arides est justifiée. L'enquête a touché 14000 dromadaires représentant 15 p. 100 des effectifs recensés dans les zones de l'étude. Les troupeaux étaient en majorité de petite taille (48 p. 100), les grands effectifs (13 p. 100) provenaient des zones du Sud du pays. Les femelles représentaient 63 p. 100 des effectifs et les jeunes 30 p. 100 .

Sur les 252 éleveurs interrogés, seuls 11 p. 100 avaient des associés. L'élevage constituait l'unique activité professionnelle des deux tiers des propriétaires. Les contraintes de cet élevage étaient liées à sa faible fécondité (un chamelon tous les 24 à 30 mois en moyenne) aggravée par une forte mortalité des jeunes, d'où une faible productivité. L'enquête rétrospective sur la mortalité des chamelons, sur trois ans, a donné un taux de mortalité moyen de 22 p. 100 en 1998 et 1997. En 1996, ce taux était de 16 p. 100 du fait des bonnes conditions pluviométriques qui ont permis l'amélioration des conditions d'alimentation des parents. Ces forts taux de mortalité étaient d'origine multifactorielle et complexe (pathologie, nutrition, environnement, pratiques d'élevage...).

La prise de colostrum par le jeune était observée dans 88 p. 100 des cas, mais 11 p. 100 des éleveurs la limitaient. Trois stratégies de sevrage étaient pratiquées par les chameliers. Le sevrage précoce, réalisé par 18 p. 100 des éleveurs, obéissait à des objectifs de vente des chamelons pour les boucheries du Sud, zone traditionnelle de consommation de produits du dromadaire. Dans la seconde stratégie, qui regroupait la majorité des troupeaux (66 p. 100), les éleveurs exploitaient le lait tout en laissant le petit téter une partie de la production pendant un an. La dernière stratégie, adoptée par 14 p. 100 des éleveurs, consistait à laisser le chamelon librement avec sa mère ; le sevrage était en général naturel, l'éleveur n'intervenant qu'au cas où les tétées se poursuivaient après fécondation de la chamelle ; le chmel était alors utilisé dans 80 p. 100 des cas.

La supplémentation alimentaire n'était pratiquée qu'occasionnellement, en année de sécheresse (84 p. 100), mais elle pouvait être poursuivie en cas de mauvaises années successives. Les pratiques sanitaires étaient observées, même si la disponibilité des produits pharmaceutiques n'était pas toujours assurée ; 65 p. 100 des éleveurs pratiquaient le déparasitage externe de leurs animaux occasionnellement, 32 p. 100 le faisaient régulièrement. Les pratiques de vermifugation étaient rares (26 p. 100), peut-être en raison des conditions d'aridité de la zone qui ne permettent pas de grosses infestations parasitaires.

L'analyse multivariée a mis en évidence cinq classes de pratiques d'élevage. La première classe comportait 40 troupeaux. Elle était caractérisée par une stratégie de sevrage à un an (82 p. 100), la traite partielle des femelles (95 p. 100), la prise de colostrum limitée $(62$ p. 100) et une période de tétée volontaire observée par 75 p. 100 des éleveurs. La classe 2 contenait 44 troupeaux dont 75 p. 100 étaient de petite taille. Elle était caractérisée par une stratégie de sevrage précoce (98 p. 100). La classe 3 contenait 20 p. 100 des troupeaux. Elle était caractérisée par une stratégie de sevrage à un an pour l'ensemble des jeunes, la traite n'était pratiquée qu'en bonne année. La classe 4 comportait 16 p. 100 de grands troupeaux ; les femelles en lactation étaient traites partiellement, l'âge au sevrage dépassait un an. La classe 5 était composée de 62 troupeaux dont 8 p. 100 de grande taille ; c'est la classe où la vermifugation était la plus courante.

La stratégie de déplacement a montré une tendance à la sédentarisation ou aux déplacements de faible amplitude dans la province pour des raisons de sécurité alimentaire et pathologique. La majorité des éleveurs interrogés restaient sur place toute l'année (53 p. 100), 29 p. 100 effectuaient plusieurs petits déplacements et 18 p. 100 de grands déplacements. Ces stratégies correspondaient aux différentes tailles des troupeaux identifiés : les grands déplacements étaient en général effectués par les grands troupeaux des provinces sahariennes qui disposaient de grandes étendues de parcours naturels, alors que les deux autres stratégies étaient adoptées par les petits et moyens troupeaux dont les déplacements étaient limités. Toutes ces stratégies pouvaient être profondément remises en cause en cas de déficits fourragers importants.

L'analyse du profil sanitaire des dromadaires adultes a montré trois classes. La première, composée de 26 troupeaux originaires pour l'essentiel des provinces d'Errachidia et de Tata, était caractérisée 
par la présence forte de la trypanosomose (72 p. 100 des cas recensés) et un taux de mortalité important sur 42 p. 100 des troupeaux. La classe 2 contenait 36 troupeaux des provinces de Laâyoune et de Guelmim, chez lesquels les maladies dominantes des adultes étaient la variole (28 p. 100) et l'ectoparasitose. La classe 3 était composée de 61 troupeaux des provinces de Zagora, d'Essaouira et d'Errachidia, où dominait le groupe « autres pathologies », avec le plus fort taux de mortalité des chamelons (39 p. 100). La classe 4 contenait 50 p. 100 des troupeaux suivis, originaires des provinces de Laâyoune et d'Essaouira ; les gros troupeaux représentaient 12 p. 100 de cette classe où l'on rencontrait l'ensemble des grandes pathologies du système d'élevage camelin et des taux de mortalités forts.

Le test du $\mathrm{Chi}^{2}$ a montré le lien entre l'état sanitaire des parents et l'apparition de la mortalité chez leurs petits, notamment certaines maladies dont la présence signe les pertes importantes de jeunes (syndromes respiratoires et « autres pathologies »). D'autres maladies comme les parasitoses externes (gale, teigne) et la variole étaient faiblement corrélées avec la mortalité des jeunes. La comparaison des fréquences d'apparition de la mortalité a montré que d'autres pathologies des adultes influaient sur la mortalité du chamelon, même si cette influence n'a pas paru énorme dans les résultats obtenus (trypanosomose).

Le test du $\mathrm{Chi}^{2}$ a démontré également les corrélations entre certaines pratiques et la mortalité des jeunes. Le déparasitage externe était corrélé avec la mortalité des chamelons, la fréquence des taux de mortalité forts allait de 36 p. 100 pour les animaux déparasités à 46 p. 100 pour les élevages qui n'observaient pas cette pratique. Des pratiques comme la prise de colostrum, la vermifugation et la vaccination jouaient également un rôle dans l'apparition des mortalités, même si ce rôle n'a pas été nettement mis en évidence par les résultats de l'enquête. L'hypothèse de la corrélation de la prise de colostrum avec l'apparition des mortalités n'a pu être confirmée entièrement par l'étude. Cependant, l'examen des fréquences de mortalité a montré que la privation de colostrum avait une part de responsabilité dans les forts taux de mortalité des jeunes. Là aussi, la formulation des questions pouvait avoir semé la confusion dans l'esprit de l'enquêteur. Bien que la prise du colostrum pendant quatre à six jours ait été une règle dans tous les élevages, il était difficile de s'en assurer pour chaque individu, surtout dans les grands troupeaux et en particulier pour les chamelles primipares « difficiles » (non traites). L'état sanitaire et l'état nutritionnel des chamelons étaient des facteurs importants dans l'apparition des mortalités. Les diarrhées étaient la principale cause de mortalité identifiée chez les chamelons sur l'ensemble de la zone d'étude, ce

\section{BIBLIO GRAPH IE}

1. BENGOU MI M., BERRADA J., TLIGUI N.S., 1997. Etude des causes de mortalité du chamelon dans le Sud du Maroc. Note bibliographique et méthodologique. Rapport projet ACSAD. Rabat, Maroc, Institut agronomique et vétérinaire.

2. BENGOUMI M., MICHEL J.F., HIDANE K., BONNET P., FAYE B., 1998. Ecopathological study of the camel young mortality in the South of Morocco. In: Proc. 3rd Annual meeting for animal production under arid conditions, Al-Ain, UAE, 2-3 M ay 1998.

3. BURGMEISTER R., 1975. Problèmes posés par l'élevage de chameaux en Afrique : exemple du Sud tunisien. Schreihe Gesellsch. Tech. Zu Arb., 21.

4. COSSIN S N., 1971. Pastoralism under pressure: a study of the Somali classes in the Jijiga area of Ethiopia. Addis Abeba, Ethiopia, Livestock and $M$ eat Board, $101 \mathrm{p}$. qui rejoint les travaux antérieurs $(1,5,8,9)$. Les insuffisances alimentaires jouaient également un rôle non négligeable dans cette mortalité. La forte homogénéité observée dans l'organisation des élevages, les déplacements et les pratiques d'élevage (surveillance des femelles pendant la mise bas, prise de colostrum, sevrage, allottement, supplémentation, traitement et prévention des maladies...) n'a pas permis de mettre en évidence une relation nette entre la mortalité et ces facteurs. En effet, la majorité des élevages enquêtés étaient conduits d'une manière plus ou moins identique. La différence avec les travaux réalisés à Laâyoune $(2,8)$, qui ont montré un effet important du mode de conduite et de gestion du troupeau sur la mortalité, serait liée à l'existence de quelques élevages « pépiniéristes » (produisant des géniteurs et subventionnés par l'Etat), ou bien périurbains, qui ont une conduite particulière.

L'influence de l'état sanitaire des parents sur la mortalité des chamelons confirme ainsi les hypothèses de départ. Le test $\mathrm{du} \mathrm{Chi}^{2} \mathrm{a}$ montré en effet des corrélations fortes entre certaines affections des adultes et les forts taux de mortalité observés chez les chamelons (syndromes respiratoires, « autres pathologies »). D'autres affections avaient des corrélations moins nettes avec cette mortalité (parasitoses, variole). Les pratiques de soins sanitaires sur les adultes influençaient l'apparition des mortalités périnatales, ce qui confirme les premiers résultats.

\section{CONCLUSION}

Les causes de mortalité avancées par les éleveurs ont confirmé la responsabilité des diarrhées (70 p. 100 des premières causes) dans l'ensemble de la zone de l'étude, puis celle des insuffisances alimentaires. Les autres causes identifiées étaient les abcès, les mortinatalités et « autres pathologies » dont l'importance variait d'une province à une autre. Des problèmes d'intoxication par les plantes ont été évoqués par les éleveurs des provinces de Zagora et de Tata, liés certainement à la mise précoce du chamelon sur parcours.

Les axes de recherche identifiés ne sauraient être traités efficacement par de seules enquêtes ponctuelles comme celle-ci, mais plutôt par la mise en place de véritables suivis d'élevages représentatifs, dans l'ensemble des provinces concernées par le problème. Des études plus approfondies (étiologie, physiopathologie, traitement, prévention...) sur les principales causes de la mortalité des chamelons - notamment les diarrhées, les abcès et les maladies respiratoires - permettraient de diminuer cette mortalité et d'améliorer la rentabilité de l'élevage camelin.

5. FAYE B., 1997. Le guide de l'élevage du dromadaire. Libourne, France, Sanofi, $126 \mathrm{p}$.

6. MICHEL J.F., BENGOUMI M., BONNET P., HIDANE K., ZRO K., FAYE B., 1997. Typologie des systèmes de production camelins dans la province de Laâyoune, Maroc. Revue Elev. Méd. vét. Pays trop., $50: 313-323$

7. RICHARD D., 1975. Etude de la pathologie du dromadaire dans la sous-province du Borana (Ethiopie). Thèse Méd. vét., Ecole nationale vétérinaire, Maisons-Alfort, France, $181 \mathrm{p}$.

8. RICHARD D., PLANCHENAULT D., GIOVANNETTI J.F., 1985. Projet de développement de l'élevage dans le Niger Centre-Est. Productions camelines (Rapport final). M aisons-Alfort, France, lemvt, 135 p.

9. SALEY M., 1990. Performances de reproduction du dromadaire au Niger. In: Proc. Workshop it is possible to improve reproductive performances in the camel, Paris, France, 10-12 September 1990. Maisons-Alfort, France, lemvt, p. 379-386.

10. WILSO N R.T., 1984. The camel. London, UK, Longman, 223 p. 


\section{Summary}

Bengoumi M., Gandega E.B., El Abrak A., Berrada J., Faye B. Study on camel calf mortality in South Morocco: Retrospective survey

Between January and August 1999 a retrospective survey was conducted in the provinces of Oued Eddahab, Laayoune, Guelmim, Tata, Essaouira, Zagora, O uarzazate, Errachidia and Figuig on 252 camel herds, i.e. 15\% of the counted populations in these areas. Calf perinatal mortality remains a major constraint to development of camel husbandry with on average $20.2 \%$ deaths (16.4\% in 1996 and $22.9 \%$ in 1997). In more than $80 \%$ cases death occurred between 0 and 6 months of age. Main mortality causes reported by breeders were diarrhea $(72 \%)$, then far behind feed insufficiency $(9 \%)$, stillbirth $(6 \%)$ and abscesses (5\%), with wide variations among provinces. Herd typology helped identify four classes based on health status and five based on livestock practices. Strong correlations between camel calf mortality and some adult camel diseases, notably the respiratory syndrome, camel pox, mange-moth and other diseases, were observed. Because management modes were very similar, correlations between mortality and breeding practices, which probably played a major role, could not be highlighted.

Key words: Dromedary - Young animal - Mortality - Morocco.

\section{Resumen}

Bengoumi M., G andega E.B., El Abrak A., Berrada J., Faye B. Estudio de la mortalidad de los camellos jóvenes al Sur de Marruecos: encuesta retrospectiva

La encuesta retrospectiva se realizó entre enero y agosto 1999, en las provincias de Oued Eddahab, Laâyoune, Guelmim, Tata, Essaouira, Zagora, O uarzazate, Errachidia y Figuig. Incluyó 252 hatos de camellos, o sea $15 \%$ del efectivo incluido en el censo de estas regiones. La mortalidad perinatal del camello joven sigue siendo un obstáculo mayor para el desarrollo de la crianza de camellos y representa un promedio de $20,2 \%$ (16,4 \% en 1996 y 22,9\% en 1997). En más de $80 \%$ de los casos, la edad de la muerte se sitúa entre 0 y 6 meses de edad. Las primeras causas de mortalidad citadas por los criadores son diarrea (72\%), siguiendo de lejos la insuficiencia alimenticia (9\%), natimuertos (6\%) y abscesos $(5 \%)$, con fuertes variaciones según las provincias. La tipología de los establecimientos permitió distinguir quatro clases según el perfil sanitario y cinco clases según las prácticas de crianza. Existen fuertes correlaciones entre la mortalidad de los camellos jóvenes y ciertas patologías de los dromedarios adultos, sobre todo el síndrome respiratorio, la viruela, la sarna y otras patologías. La fuerte homogeneidad de las formas de conducta no permitió poner en evidencia correlaciones entre la mortalidad y las prácticas de manejo, que juegan probablemente un importante papel.

Palabras clave: Dromedario - Animal joven - Mortalidad Marruecos. 